RESENDE, G.M.; FLORI, J.E. Rendimento e qualidade de cultivares de pepino para processamento em função do espaçamento de plantio. Horticultura Brasileira, Brasília, v.22, n.1, p. 117-120, jan-mar 2004.

\title{
Rendimento e qualidade de cultivares de pepino para processamento em função do espaçamento de plantio
}

\author{
Geraldo M. de Resende; José Egidio Flori \\ Embrapa Semi-Árido, C. Postal 23, 56300-000 Petrolina-PE; E-mail: gmilanez@ufla.br
}

\begin{abstract}
RESUMO
Avaliou-se o rendimento e a qualidade de cultivares de pepino para processamento em diferentes espaçamentos de plantio, em experimento em Petrolina (PE), de abril a julho/99. O delineamento experimental utilizado foi de blocos casualizados sendo os tratamentos dispostos no esquema fatorial $5 \times 3$, compreendendo cinco cultivares (Calypso, Eureka, Supremo, Vlaspik e Vlasset) e três espaçamentos entre plantas $(0,20 ; 0,30$ e $0,50 \mathrm{~m})$ com três repetições. A parcela experimental foi composta de quatro linhas de 3,0 m espaçadas de 1,00 m, com duas plantas por cova, sendo as duas linhas centrais consideradas como área útil. Verificou-se efeitos lineares negativos na produtividade com o incremento do espaçamento entre plantas para as cultivares Vlaspik, Vlasset e Eureka, e efeitos quadráticos com pontos de mínima e máxima produtividade para as cultivares Calypso e Supremo. As maiores massas frescas de frutos foram observadas nas cultivares Vlaspik $(35,44 \mathrm{~g} /$ fruto) e Vlasset $(35,07 \mathrm{~g} /$ fruto) que não apresentaram diferenças entre si. Observou-se efeito linear positivo com o aumento do espaçamento entre plantas no número de frutos por planta para as cultivares Vlaspik, Vlasset e Supremo. Para as cultivares Calypso e Eureka constataram-se efeitos quadráticos com pontos de máximo número de frutos por planta. Na classificação de frutos comerciais não verificou-se efeitos significativos dos tratamentos.
\end{abstract}

Palavras-chave: Cucumis sativus, classificação, produtividade, densidade.

\begin{abstract}
Effect of plant spacing on the yield and quality of pickling cucumber cultivars

The yield and quality of pickling cucumber cultivars was evaluated in Petrolina, Pernambuco State, Brazil, from April to June of 1999. The experimental design was a complete randomized block, with three replications in a $5 \times 3$ factorial scheme, with five cultivars (Calypso, Eureka, Supremo, Vlaspik and Vlasset), and three planting spaces $(0.20 ; 0.30$ and $0.50 \mathrm{~m})$. Experimental plots consisted of four rows spaced $1.00 \mathrm{~m}$ apart, with three meters in length and two plants per hole. The main plot was comprised of the two central rows. Negative linear effect on yield was observed with the increase of the interplant spacing (inside the row) for the cultivars Vlaspik, Vlasset and Eureka and the cultivars Supremo and Calypso showing quadratic effects points of minimum and maximum. The greatest fresh mass of fruits was found on the cultivars Vlaspik ( $35.44 \mathrm{~g} /$ fruit) and Vlasset ( $35.07 \mathrm{~g} /$ fruit) with no statistical differences between them. Positive linear effect for the number of fruits per plant was observed with increasing interplant spacing for the cultivars Vlaspik, Vlasset and Supremo. Quadratic effect was observed in 'Calypso' and 'Eureka' with maximum points of the number of fruits per plant. No significant effect of the treatments was observed for the commercial fruit production.
\end{abstract}

Keywords: Cucumis sativus, grading, yield, density.

(Recebido para publicação em 4 de abril de 2003 e aceito em 22 de outubro de 2003)

$\mathrm{A}$ evolução observada na olericultura brasileira nos últimos anos mostra tendência ao aumento do consumo de produtos processados com maior valor agregado. Dentre as hortaliças consumidas em conserva, o pepino é uma das mais importantes (Costa, 2000). A cultura do pepino para processamento ocupa posição de destaque na região Sul do Brasil, tendo o estado de Santa Catarina como principal produtor nacional (Silva et al., 1992). Nesta região, o cultivo desta olerácea para produção de frutos destinados à industrialização é realizado, empregando-se híbridos ginóicos com elevado potencial de rendimento de frutos (Espinola, 2000). Contudo, sendo o pepino uma cultura que requer grande intensidade de luz e longo período de temperatura elevada, tendo pouca resistência ao frio (Whitaker e Davis, 1962; Knott, 1966), o período de entressafra no inverno das regiões Sul e Sudeste é uma realidade que ocasiona queda na produção e ociosidade da indústria (Nadal et al., 1986). Neste contexto, os perímetros irrigados da região Nordeste e do Norte de Minas Gerais, emergem com grande potencial de produção durante todo o ano, em função de suas condições climáticas.

A população ideal de plantas a ser empregada é aquela suficiente para atingir o índice de área foliar (IAF) ótimo a fim de interceptar o máximo de radiação solar útil à fotossíntese e ao mesmo tempo maximizar a fração da matéria seca alocada para os frutos. A população de plantas afeta a penetração da radiação solar no dossel e o equilíbrio entre o crescimento das partes vegetativas e dos frutos. Modificações na eficiência das fontes, através de uma alteração na popula- ção de plantas ou do aumento da disponibilidade de radiação, afetam indiretamente a distribuição da matéria seca entre os órgãos da planta. Esse efeito ocorre de forma indireta, mediante alterações no número de frutos em crescimento, os quais modificam a capacidade de dreno da planta (Marcelis, 1993; Hao e Papadopoulos, 1999).

O manejo da cultura do pepino para conserva tem como objetivo principal maximizar o rendimento de frutos. Entretanto, a produção de frutos depende diretamente do compartimento vegetativo da planta, que é responsável pela produção dos assimilados. Para maximizar a produção de frutos é necessário, portanto, atingir a produção potencial de assimilados em nível da planta inteira e em seguida alocar para os frutos a maior fração possível desses 
assimilados. Uma das formas de manejo da produção e da distribuição dos assimilados é através da população de plantas e da poda (Schvambach et al., 2002).

Hughes et al. (1974) citam que o espaçamento mais comum para colheitas manuais é de 1,00 m entre fileiras e 0,15 a $0,20 \mathrm{~m}$ entre plantas, o que corresponde a populações de 50.000 a 66.666 plantas/ha. Resende (1999) na densidade de 66.666 plantas/ha (1,00 x $0,30 \mathrm{~m}$ com duas plantas por cova), destacou como mais produtivas as cultivares Indaial $(29,72 \mathrm{t} / \mathrm{ha})$, Score $(26,46 \mathrm{t} /$ ha), Colônia (26,43 t/ha) e Ginga AG77 (26,12 t/ha). A cultivar Pérola foi a que apresentou a produtividade significativamente mais baixa $(13,91 \mathrm{t} / \mathrm{ha})$. A massa fresca do fruto e o número de frutos por planta variaram de 49,2 g ('PrimepaK') a 67,4 ('Pérola') e 3,2 ('Pérola') a 7,7 frutos/planta ('Indaial'), respectivamente.

No trabalho de Resende e Flori (2003) a produtividade comercial de frutos variou de 21,43 a $34,54 \mathrm{t} / \mathrm{ha}$, enquanto a maior massa fresca de frutos foi obtida pela cultivar Francipak (37,79 g/ fruto), não diferindo estatisticamente das cultivares Flurry, Vlaspik, Vlasstar, Eureka, Ginga AG-77, Supremo e Navigator (34,05 a 36,24 g/fruto). A cultivar Eureka apresentou maior número de frutos por planta (14,81 frutos/ planta), sem diferir estatisticamente das cultivares Calypso, Primepak, Vlasset, Fancipak, Imperial, Ginga AG-77, Flurry e Panorama. Para classificação de frutos comerciais, verificou-se uma maior proporção de frutos tipo 1 , tendo todas as cultivares apresentado valores superiores a $85 \%$.

Estudando diferentes espaçamentos entre plantas $(0,75 \mathrm{~m}$ x 0,20; 0,30 e 0,40 $\mathrm{m})$, Gebologlu e Sagllam (2002), verificaram maiores produtividades no espaçamento com $0,20 \mathrm{~m}$ entre plantas, enquanto Peil e Lopez-Galvez (2002) observaram um decréscimo no número e na biomassa dos frutos com o aumento da densidade de plantio. O efeito da densidade de plantio foi maior em termos de matéria seca do que na matéria fresca do fruto.

Não há muitas informações sobre o plantio de pepino para processamento nas condições do Vale do são Francisco e neste contexto, o presente trabalho objetivou determinar o efeito da população de plantas sobre cultivares de pepino para processamento, visando maior rendimento e qualidade de frutos, como uma nova alternativa agroindustrial para a região.

\section{MATERIAL E MÉTODOS}

O experimento foi conduzido no Campo Experimental de Bebedouro, Embrapa Semi-Árido, Petrolina, de abril a julho de 1999, cujas coordenadas geográficas são $9^{\circ} 9^{\prime}$ de latitude Sul e $40^{\circ}$ 29' de longitude Oeste e altitude de 365,5 m em Latossolo Vermelho Amarelo com as seguintes características: $\mathrm{pH}$ $\left(\mathrm{H}_{2} \mathrm{O}\right)=6,4 ; \mathrm{Ca}^{2+}=2,4 \mathrm{cmol} / \mathrm{dm}^{3} ; \mathrm{Mg}^{2+}$ $=0,7 \mathrm{cmol} / \mathrm{dm}^{3} ; \mathrm{Na}^{+}=0,04 \mathrm{cmol}_{\mathrm{c}} / \mathrm{dm}^{3}$; $\mathrm{K}^{+}=0,35 \mathrm{cmol} / \mathrm{dm}^{3} ; \mathrm{Al}^{3}=0,04 \mathrm{cmol}_{\mathrm{c}} /$ $\mathrm{dm}^{3}$ e P/Mehlich $=24,78 \mathrm{mg} / \mathrm{L}$. O delineamento experimental utilizado foi de blocos casualizados sendo os tratamentos dispostos em esquema fatorial $5 \times 3$ compreendendo cinco cultivares (Calypso, Eureka, Supremo, Vlaspik e Vlasset) e três espaçamentos entre plantas $(0,20 ; 0,30$ e $0,50 \mathrm{~m})$ em três repetições. As parcelas experimentais constaram de quatro linhas de 3,0 m espaçadas de $1,00 \mathrm{~m}$, com duas plantas por cova, sendo as duas linhas centrais consideradas como área útil.

$\mathrm{Na}$ adubação de plantio, feita com base na análise de solo, utilizou-se 150 $\mathrm{kg} /$ ha de sulfato de amônio, $220 \mathrm{~kg} / \mathrm{ha}$ de superfosfato simples e $65 \mathrm{~kg} / \mathrm{ha}$ de cloreto de potássio. Também foram realizadas duas adubações em cobertura com $150 \mathrm{~kg} / \mathrm{ha}$ de sulfato de amônio, fazendo-se a primeira aos 21 dias após a semeadura (após o desbaste) e a segunda 15 dias após esta.

Foram realizadas irrigações por aspersão, três vezes por semana, sendo a cultura mantida no limpo através de capinas manuais e conduzida sob sistema rasteiro. Para controle de pragas foram utilizados deltametrina e pirimicarb, quando necessários, e para controle de doenças semanalmente foram utilizados preventivamente mancozeb e benomil, pulverizados somente até o início da floração (30 dias após a semeadura). Após este período, foram utilizados somente produtos à base de enxofre e de- tergente neutro, pulverizados semanalmente até o fim da colheita.

As colheitas foram feitas três vezes por semana, iniciando-se aos 34 dias após a semeadura e estendendo-se por um período de 30 dias. Foram avaliadas a produtividade comercial estimada ( $\mathrm{t} / \mathrm{ha})$, massa fresca de fruto $(\mathrm{g})$, e número de frutos por planta e classificação de frutos comerciais em percentagem. Foram considerados frutos comerciais os não deformados, retilíneos, sem manchas e sem lesões e estes foram classificados em classe 1(frutos com 6 a $9 \mathrm{~cm}$ de comprimento) e classe 2 (frutos com 9 a $12 \mathrm{~cm}$ de comprimento) (Resende, 1999). Os dados foram submetidos à análise de variância e as médias comparadas pelo teste de Tukey para cultivares e regressão polinomial para espaçamentos, com base no modelo quadrático, ao nível de $5 \%$ de probabilidade. Os dados originais de porcentagem foram transformados em arcoseno $\sqrt{P / 100}$ para a análise estatística, sendo apresentados nos resultados as médias originais.

\section{RESULTADOS E DISCUSSÃO}

Houve efeito significativo para a interação entre as cultivares e espaçamentos entre plantas para as diferentes características avaliadas. Verificou-se que a maior produtividade comercial foi obtida no menor espaçamento entre plantas $(0,20 \mathrm{~m})$ para todas as cultivares avaliadas, onde 'Vlaspik' destacou-se com a maior produtividade média (29,06 t/ha), seguida pela 'Vlasset' com 22,74 t/ha (Tabela 1). Utilizando a mesma densidade de plantio, Resende (1999), avaliando diferentes cultivares encontrou resultados semelhantes em termos de produtividade comercial de frutos. Para as cultivares avaliadas no presente experimento, no espaçamento de $1,00 \times 0,30 \mathrm{~m}$, Resende et al. (2001) verificaram produtividade superior variando de 30,97 a 39,78 t/ha; no entanto, em plantio realizado de setembro a novembro, sob condições de temperatura mais alta, favorável à cultura conforme relata Whitaker e Davis (1962). Estes resultados são similares às afirmações de Hughes et al. (1974) que citam ser o espaçamento de 1,00 m en- 
Tabela 1. Produtividade comercial, número de frutos por planta e por metro quadrado, massa fresca e classificação de frutos de cultivares de pepino em função de espaçamentos de plantio. Petrolina, Embrapa Semi-Árido, 1999.

\begin{tabular}{|c|c|c|c|c|}
\hline \multirow{2}{*}{ Cultivares } & \multicolumn{4}{|c|}{ Espaçamento entre plantas (m) } \\
\hline & 0,20 & 0,30 & 0,50 & \multirow{2}{*}{ Média } \\
\hline \multicolumn{2}{|c|}{ Produtividade comercial (t/ha) } & \multicolumn{2}{|c|}{ C.V. $(\%)=4,54$} & \\
\hline Vlaspik & 33,15 a & $30,19 a$ & 23,83 a & 29,06 a \\
\hline Vlasset & $24,06 \mathrm{~b}$ & $23,72 b$ & $20,43 \mathrm{~b}$ & $22,74 \mathrm{~b}$ \\
\hline Supremo & $26,41 b$ & $20,21 \mathrm{c}$ & $15,86 \mathrm{c}$ & $20,82 \mathrm{c}$ \\
\hline Eureka & $25,21 b$ & $21,43 \mathrm{bc}$ & $15,25 \mathrm{c}$ & $20,63 \mathrm{~cd}$ \\
\hline Calypso & $21,57 \mathrm{c}$ & $20,99 \mathrm{c}$ & $15,17 \mathrm{c}$ & $19,24 \mathrm{~d}$ \\
\hline Média & 26,08 & 23,31 & 18,11 & 22,50 \\
\hline \multicolumn{3}{|c|}{ Número de frutos por planta } & \multicolumn{2}{|c|}{ C.V. $(\%)=3,46$} \\
\hline Vlaspik & $9,69 a$ & $12,56 \mathrm{a}$ & $16,52 \mathrm{a}$ & $12,92 \mathrm{a}$ \\
\hline Vlasset & 7,16 bc & $10,58 b$ & $15,19 b$ & $10,98 \mathrm{~b}$ \\
\hline Supremo & $7,74 \mathrm{~b}$ & $8,83 \mathrm{c}$ & $11,70 \mathrm{c}$ & $9,44 \mathrm{c}$ \\
\hline Calypso & $6,50 \mathrm{c}$ & $9,28 \mathrm{c}$ & $11,19 \mathrm{~cd}$ & $8,99 \mathrm{c}$ \\
\hline Eureka & $7,21 \mathrm{bc}$ & $9,18 \mathrm{c}$ & $10,72 \mathrm{~d}$ & $9,04 \mathrm{c}$ \\
\hline Média & 7,66 & 10,09 & 13,06 & 10,27 \\
\hline \multicolumn{3}{|c|}{ Número de frutos por metro quadrado } & \multicolumn{2}{|c|}{ C.V. $(\%)=3,02$} \\
\hline Vlaspik & 96,62 a & 83,72 a & $66,08 \mathrm{a}$ & $82,14 \mathrm{a}$ \\
\hline Vlasset & $71,56 \mathrm{~b}$ & $70,50 \mathrm{~b}$ & $60,94 \mathrm{~b}$ & $67,67 \mathrm{~b}$ \\
\hline Supremo & $75,67 \mathrm{~b}$ & $59,22 \mathrm{c}$ & $46,78 \mathrm{c}$ & $60,56 \mathrm{c}$ \\
\hline Calypso & $65,00 \mathrm{c}$ & $61,89 \mathrm{c}$ & $44,78 \mathrm{c}$ & $57,22 \mathrm{~cd}$ \\
\hline Eureka & $72,11 b$ & $61,22 \mathrm{c}$ & $42,89 \mathrm{c}$ & $58,74 \mathrm{~d}$ \\
\hline Média & 76,19 & 67,31 & 52,29 & 65,27 \\
\hline \multirow{2}{*}{\multicolumn{2}{|c|}{ Massa fresca dos frutos $(\mathrm{g})$}} & & \multicolumn{2}{|c|}{ Classificação de frutos (\%) } \\
\hline & & \multicolumn{2}{|c|}{ Classe 1} & Classe 2 \\
\hline Vlaspik & 35,44 a & \multicolumn{2}{|c|}{91,93 a } & $8,07 \mathrm{a}$ \\
\hline Vlasset & 35,07 a & \multicolumn{2}{|c|}{93,81 a } & $6,19 a$ \\
\hline Supremo & 33,99 a & \multicolumn{2}{|c|}{92,29 a } & $7,71 \mathrm{a}$ \\
\hline Calypso & 33,61 a & \multicolumn{2}{|c|}{92,27 a } & $7,73 \mathrm{a}$ \\
\hline Eureka & $33,59 \mathrm{a}$ & \multicolumn{2}{|c|}{$91,94 \mathrm{a}$} & $8,06 \mathrm{a}$ \\
\hline$\overline{\text { C.V. }}$ & 4,43 & \multicolumn{2}{|c|}{3,03} & 14,49 \\
\hline
\end{tabular}

Médias seguidas de mesma letra minúscula e maiúscula nas colunas não diferem entre si a $5 \%$ de probabilidade pelo teste de Tukey.

tre fileiras e 0,15 a $0,20 \mathrm{~m}$ entre plantas o mais comum para colheitas manuais e com Gebologlu e Sagllam (2002), que avaliando diferentes espaçamentos entre plantas $(0,20 ; 0,30$ e $0,40 \mathrm{~m})$, verificaram maiores produtividades no espaçamento com $0,20 \mathrm{~m}$ entre plantas.

Verifica-se pela Tabela 1, com o aumento do espaçamento entre plantas, maior número de frutos por planta em todas as cultivares. As cultivares Vlaspik e Vlasset sobressaíram-se com maior número médio de frutos por planta (12,92 e 10,98 respectivamente).
Resende et al. (2001) encontraram valores oscilando entre 10,68 a 13,90 frutos/planta para as cultivares avaliadas no espaçamento de $1,00 \times 0,30 \mathrm{~m}$, enquanto Resende e Flori (2003) obtiveram de 9,90 a 14,81 frutos/planta.

Verificou-se uma redução no número de frutos por metro quadrado com o incremento do espaçamento entre plantas, sendo as cultivares Vlaspik e Vlasset as que se destacaram com maiores produções médias de frutos (82,14 e 67,67 frutos $/ \mathrm{m}^{2}$, respectivamente) (Tabela 1). Estes resultados são concordantes com
Peil e Lopez-Galvez (2002) que observaram um decréscimo no número e na biomassa dos frutos com o aumento da densidade de plantio. Em cucurbitáceas, de forma geral, altas densidades produzem grande número de frutos por área, mas com tamanho, peso e número de frutos por planta reduzidos. Esse fato, segundo Robinson e Walters (1997), tem sido atribuído principalmente às pressões de competição inter e intraplantas. Nas baixas densidades, tem sido verificado o inverso, ou seja, produção total menor com maior número de frutos por 
planta, de tamanho e peso mais elevado. As pressões exercidas pela população de plantas afetam de modo marcante o seu desenvolvimento. Quando a densidade de plantas aumenta por unidade de área, atinge-se um ponto no qual as plantas competem por fatores essenciais de crescimento, como nutrientes, luz e água (Janick, 1968).

Não foram observadas diferenças significativas entre os tratamentos, para massa fresca do fruto (Tabela 1). No entanto, verificou-se que as maiores massas frescas de frutos foram apresentadas pelas cultivares Vlaspik $(35,44 \mathrm{~g} /$ fruto) e Vlasset (35,07 g/fruto). Resultados semelhantes são relatados por Resende e Flori (2003).

Não se verificou efeitos significativos dos tratamentos na produção de frutos classe 1 (variação de 91,92 a 93,81\% entre as cultivares) e classe 2 (variação de 6,18 a $8,07 \%$ entre as cultivares). Resende e Flori (2003), utilizando o espaçamento de $1,00 \times 0,30 \mathrm{~m}$, também verificaram maior proporção de frutos classe 1, nas mesmas cultivares estudadas, com variações de 85,83 a 92,21\%.

Em função dos resultados obtidos, verificou-se que o espaçamento de 0,20 m entre plantas é o mais indicado para o cultivo de pepino para processamento no Vale do São Francisco para todas as cultivares avaliadas e que as cultivares Vlaspik e Vlasset se destacaram com as maiores produtividades.

\section{LITERATURA CITADA}

COSTA, C.P. Olericultura Brasileira: passado, presente e futuro. Horticultura Brasileira, Brasília, v.18, Suplemento, p.7-11, 2000. Conferência apresentada no $40^{\circ}$ Congresso Brasileiro de Olericultura, 2000.

ESPINOLA, H.N.R. Efeito de três doses de nutrientes minerais sobre a acumulação de massa seca e nitrogênio pela planta de pepino tipo conserva. 2000.52 p. (Tese mestrado), UFSM, Santa Maria.

GEBOLOGLU, N.; SAGLLAM, N. The effect of different plant spacing and mulching materials on the yield and fruit quality of pickling cucumber. Acta Horticulturae, v.579, p.603-607, 2002.

HAO, X.; PAPADOPOULOS, A.P. Effects of supplemental lighting and cover materials on growth, photosynthesis, biomass partitioning, early yield and quality of greenhouse cucumber. Scientia Horticulturae, v.80, n.1-2, p.1-18, 1999. HUGHES, G.R.; AVERRE, C.W.; SORENSEN, K.A. Growing pickling cucumbers in North Carolina. North Carolina: Agricultural Extension Service, $1974.27 \mathrm{p}$.

JANICK, J. A ciência de horticultura. 2.ed. Rio de Janeiro: Freitas Bastos, 1968. 485 p.

KNOTT, J.E. Handbook for vegatable growers. New York: John Wiley, 1966. 245 p.

NADAL, R.; GUIMARÂES, D.R.; BIASI, J.; PINHEIRO, S.L.G.; CARDOSO, V.I.M. Olericultura em Santa Catarina: aspectos técnicos e econômicos. Florianópolis: EMPASC, 1986. $187 \mathrm{p}$.
MARCELIS, L.F.M. Fruit growth and biomass allocation to the fruits in cucumber. 2. Effect of irradiance. Scientia Horticulturae, v.54, n.2, p.123130, 1993.

PEIL, R.M.; LOPÉZ-GALVÉZ, J. Fruit growth and biomass allocation to the fruits in cucumber: effect of plant density and arrangement. Acta Horticulturae, v.588, p.75-80, 2002.

RESENDE, G.M. Produção de pepino para conserva na região norte de Minas Gerais. Horticultura Brasileira, Brasília, v.17, n.1, p.5760, 1999.

RESENDE, G.M.; COSTA, N.D.; FLORI, J.E. Produção de pepino para conserva no Vale do São Francisco. Horticultura Brasileira, Brasília, v.19, n.2, 2001. Suplemento, CD-ROM. Trabalho apresentado no $41^{\circ}$ Congresso Brasileiro de Olericultura, 2001.

RESENDE, G.M.; FLORI, J.E. Produtividade de pepino para processamento no Vale do São Francisco. Pesquisa Agropecuária Brasileira, Brasília, v.38, n.2, p.251-255, 2003.

ROBINSON, R.W.; WALTERS, D.S.D. Cucurbits. New York: CAB International, 1997. $226 \mathrm{p}$.

SCHVAMBACH, J.L.; ANDRIOLO, J.L.; HELDWEIN, A.B. Produção e distribuição da matéria seca do pepino para conserva em diferentes populações de plantas. Ciência Rural, Santa Maria, v.32, n.1, p.35-41, 2002.

SILVA, A.C.F.; AGOSTINI, I.; MULLER, J.J.V.; VIZZOTO, V.J. Efeito de densidades populacionais sobre a produtividade de pepino para conserva. Horticultura Brasileira, Brasília, v.10, n.1, p.28-29, 1992.

WHITAKER, T.W.; DAVIS, G.N. Cucurbits: botany, cultivation and utilization. London: Leonard Hill, 1962. 250 p. 